\title{
Thermal and water transfer in cementitious porous medium: thermal building and durability
}

\author{
Xiaoyan $\mathrm{Ma}^{1, *}$, Farid Benboudjema ${ }^{1}$, and Rachid Bennacer ${ }^{1,2}$ \\ ${ }^{1}$ LMT / ENS Cachan / CNRS / Paris-Saclay University, 61 avenue du Président Wilson, 94235 Cachan, France \\ ${ }^{2}$ EPMI, F-95092 Cergy Pontoise, France
}

\begin{abstract}
Cementitious materials are typical porous medium, which are widely used in civil engineering and thermal buildings. The thermal and hydrate properties of the material are fundamental to predict accurately the energy needs and the reliable duration of the structures in a long term. Due to the complicated coupling, the thermal capacity, conductivity and source term in energy equation are affected by the humidity distribution and the microstructure evolution of the porous materials. The coupling relation of thermal and hydrate properties are summarized in this work, the coupled drying model is discussed, and the experiment is performed to verify the simulation results for RH (Relative humidity) and ML (Mass loss). Two approaches are adopted to estimate the humidity/water content profile in the porous medium and to analyse the temperature effect. In addition, the equivalent hydrate capacity and conductivity are identified as function of humidity. The significance of this work lies in predicting the energy demand, the long-term thermal behaviour, taking into account the change of the thermal cementitious materials properties during their service time. Such inner structure is consequence of the system durability where the temperature, water content and humidity will act on the cracks settling within the material.
\end{abstract}

\section{Nomenclature}

$\begin{array}{cl}c & \text { Heat capacity } \\ D_{e f f} & \text { Effective diffusivity }\left[\mathrm{m}^{2} / \mathrm{s}\right] \\ k_{l a} & \text { Relative permeability } \\ \mathrm{q} & \text { Source term } \\ L_{v} & \text { Latent heat }[\mathrm{J} / \mathrm{kg}] \\ M & \text { Location at }(\mathrm{x}, \mathrm{y}, \mathrm{z}) \\ M_{v} & \text { Molecular mass }[\mathrm{kg} / \mathrm{mol}] \\ p & \text { Pressure }[\mathrm{Pa}] \\ R H & \text { Relative humidity } \\ S & \text { Saturation degree } \\ \Phi & \text { Porosity [\%] } \\ \rho & \left.\text { Density [ } \mathrm{kg} / \mathrm{m}^{3}\right] \\ \mu & \text { Dynamic viscosity }[\mathrm{Pa} \cdot \mathrm{s}] \\ \lambda & \text { Heat conductivity }[\mathrm{W} / \mathrm{m} \cdot \mathrm{K})] \\ \mu & \text { Dynamic viscosity }[\mathrm{Pa} \cdot \mathrm{s}]\end{array}$

Sub/superscript

$\begin{array}{cl}v s & \text { Saturation vapour } \\ \text { app } & \text { Apparent } \\ l & \text { Liquid water } \\ v & \text { Water vapour }\end{array}$

*Corresponding author: ma@lmt.ens-cachan.fr

\section{Introduction}

The performance of thermal building is dependent on the properties of wall materials and their evolution overtime. The properties control is a key point in predicting accurately the energy needs over the time. Such cementitious materials are typical kind of complex porous medium, which are widely used in civil engineering. The building walls are subjected to various ambient conditions. During the lifetime of building concrete structure, some undesirable pathologies will appear after a long induction period, and eventually resulting in irreversible structure damages [1]. The most important factors that induce such structure changes (pathologies) are surrounding temperature and humidity.

Therefore, to understand the thermal properties which are related to the humidity and water contents and structure change, we have to be accurate and master the mechanisms of moisture transport inside the structure. However, the difficulties lie in the complexity of cementitious porous media, the strong coupling between temperature effect and humidity/water sorption, and the inaccurate energy source term induced by the phase change (evaporation/condensation process).

First of all, thermal conductivity as the main heat transfer parameter is necessary and often subject of measurement for various types of cement-based 
composites. However, very rare reference quantify the dynamic dependence of thermal conductivity on moisture and water content. It is complex coupling depending on the strong interlink between temperature and humidity conditions and the diffusion phenomena. The coupling phenomena also includes local aspect as total pore volume, pore distribution and connectivity. The porous structure (cementitious material) thermal conductivity varies in a very significant way due to the difference in values between air and cement stone (depending on the amount and the type of aggregates). In common service conditions, cementitious composites always contain certain amount of water. The thermal conductivity of water is $0.6 \mathrm{~W} /(\mathrm{mK})$, which is more than twenty times higher of the air. Therefore, heterogeneous water contents in the pores compete with the effect of air, so the thermal conductivity of such composite material will exhibit important change over space and between the identified values by different authors.

Pavlik and Rovnanikova measured the thermal conductivity of a cement-based composite material [2], focusing on the dependence of thermal conductivity to the moisture content from fully dry state to totally saturated state by adopting an impulse technique. Results show that the thermal conductivity increase with the increase of ambient humidity under the same temperature condition. Dell'Isola et al. demonstrated that the effective thermal conductivity of some common building materials increases with temperature and moisture content [3].

Kim et al. proposed Eq. (1) of thermal conductivity for concrete [4], deducing from the regression analysis results. In which, the aggregate volume fraction and moisture condition are revealed as the main factors affecting the concrete conductivity.

$$
\lambda_{\mathrm{R}}=0.8 \times[1.62-1.54 \times(\mathrm{W} / \mathrm{C})]+0.2 \times \mathrm{RH}
$$

In which, $\lambda_{\mathrm{R}}$ is a modification index associated with average relative humidity $(R H)$ and water-to-cement ratio $(\mathrm{W} / \mathrm{C})$. Bristow provided equivalent reliable measurements of soil thermal properties as a function of water content [5].

As consequence of change in thermal conductivity, it will modify the density and heat capacity as well. Hillel studied the thermal diffusivity varying with water content [6]. It indicated that the thermal diffusivity is small at low water contents, and it increases as water content increases, reaches a maximum, and then slowly decreases as water content continues to rise to saturation state. In addition, the thermal conductivity of humid air is also function of temperature, pressure, and mole fraction of water. Beirão et al. performed research on humid air, and it indicated that thermal conductivity increase with temperature under the same pressure for same humidity, and specific capacity of moist air increase with temperature under all humidity cases [7]. Bednarska and Koniorczyk also verified the negative influence of moisture on thermal properties of material. The values of thermal conductivity coefficient increase with the increase of liquid content in pore [8].
Another coupling of heat and mass during drying or wetting process is the local energy source term, representing the evaporation or condensation. The phase change induces the energy loss or gained accompanied with the local moisture and water contents transfer process. Such energy source term in porous medium is related to the water changing quantity. Such energy source at location $\mathrm{M}$, i.e. $\mathrm{q}(\mathrm{M})$, is the latent heat of the evaporation/condensation multiplied by the local water content change. The transient heat transfer equation with source term is given by Eq. (2).

$$
(\rho \mathrm{c})_{M} \frac{\partial \mathrm{T}}{\partial \mathrm{t}}=\nabla(\lambda(\mathrm{M}) \cdot \nabla \mathrm{T})+\mathrm{q}(\mathrm{M})
$$

Heat capacity and thermal conductivity are function of the solid material, fluid, porosity, local water content, and HR. Such inhomogeneity could be expressed as:

$(\rho c)_{M}$ or $\lambda(M) \sim$ function(porosity, water content, gas, $H R$ )

The water evaporation/condensation quantify the source term and it takes major weight because the latent heat value is $2260\left(\mathrm{~kJ} \cdot \mathrm{kg}^{-1}\right)$, which is much higher than the sensible heat $4182\left(\mathrm{~J} \cdot \mathrm{kg}^{-1} \cdot \mathrm{K}^{-1}\right)$. The question (2) could be rewritten without the $\mathrm{q}(\mathrm{M})$ term by adopting the technique of apparent heat capacity.

It is the strategy of variable heat capacity with temperature in order to incorporate the released or absorbed energy during the phase change. The partial differential equation is expressed by Eq. (3).

$$
\left(\rho C_{p}(M)\right)^{a p p} \frac{\partial \mathrm{T}}{\partial \mathrm{t}}=\nabla(\lambda(\mathrm{M}) \cdot \nabla \mathrm{T})
$$

The advantage of this method is to avoid the complexity in transform the latent heat term and simplify the calculation in source term. Therefore, to estimate this energy, we need to quantify the water that transformed in phase during the drying or wetting process. On the other hand, the drying process is quite slow in comparison to the time needed to reach temperature equilibrium. So in most studies, the drying or wetting of cementitious materials are considered as isotherm process.

In this work, aiming to obtain the local and global information of the hydrate state inside the porous material, we firstly performed the experimental trials. The inner relative humidity and the global mass loss are traced, which will be taken as the reference for the simulation results. Secondly, the analytical solution for hydrate state prediction is put forward. It is a simplified method to estimate the humidity profile because the solution is based on linear partial differential equation with constant diffusion coefficient instead of non-linear model. Afterwards the non-linear drying model for porous medium is presented and computational results for relative humidity and mass loss are obtained by CAST3M programming. Results are compared with the experimental one. At last, the equivalent diffusion coefficient and equivalent hydrate capacity is calculated as function of humidity. This information is fundamental for optimizing the hydrate and thermal properties and in the long-term predicting the durability of the cementitious materials. 


\section{Experiment and analytical solutions}

For the experiment part, the porous specimen is concrete $\mathrm{B} 11$, which has been widely studied and properties are mostly know [9]. The geometry is prismatic with size of $7 \times 7 \times 28 \mathrm{~cm}^{3}$, which is subjected to drying inside the climate chamber on two lateral faces, and the other four lateral faces are well enclosed by aluminium. The climate chamber (see Fig. 1a) is automatic regulation at temperature of $38 \pm 0.5^{\circ} \mathrm{C}$, and relative humidity of $32 \pm$ $1 \%$. The evolution of mass is recorded by balance, and humidity sensors are fixed at the preserved holes in the samples, which are $1.5,3.0$ and $6.0 \mathrm{~cm}$ to the drying surface, respectively (see arrows), as illustrated in Fig. $1 b$. The relative humidity of the fixed points are recorded continuously. Further details for the devices and protocol can be referred to previous work [10].

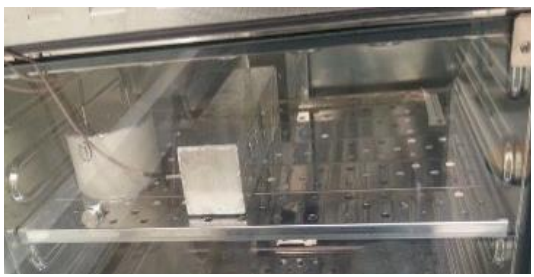

a)

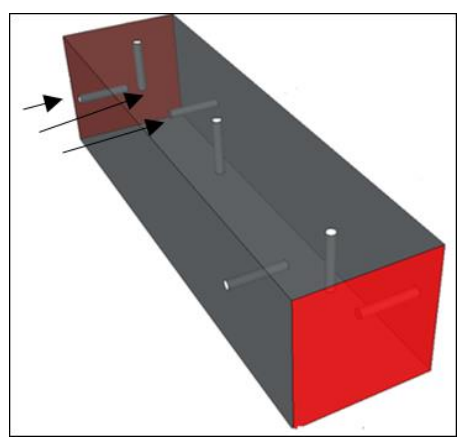

b)

Fig. 1. The climate chamber used for drying a); and prismatic concrete block with sensor locations b).

As a consequence of the pressure difference inside the domain, the complexity of the non-linear coupling of vapour diffusion and liquid water displacement feature the local phenomena. In order to have a global idea of the humidity distribution inside the porous medium, an analytical method with constant diffusion coefficient will be applied.

We will illustrate the physical tendency, to avoid the complexity by using a simplified linear model. A constant property without phase change is considered in order to use the existing analytical solution of the humidity distribution. The constant diffusion coefficient is used instead of variation function of humidity and temperature and no liquid displacement is considered. The resulting analytical solution of the internal relative humidity (RH) as function of dimensionless time and relative length are plotted in Fig. 2a. It shows the initial dimensionless humidity was unity as initial condition and the used boundary condition is zero concentration $(x=$ $1.0, \mathrm{RH}=0$ ).

The relative humidity decrease fast at the vicinity of the external boundary. The central positions (such as $x \leq$ 0.2 ) exhibit a slowly decrease over the considered time. The corresponding three experimental dimensional positions of the humidity sensors are chosen to have a clearer visualization of the local humidity time evolution, as showed in Fig. $2 b$.

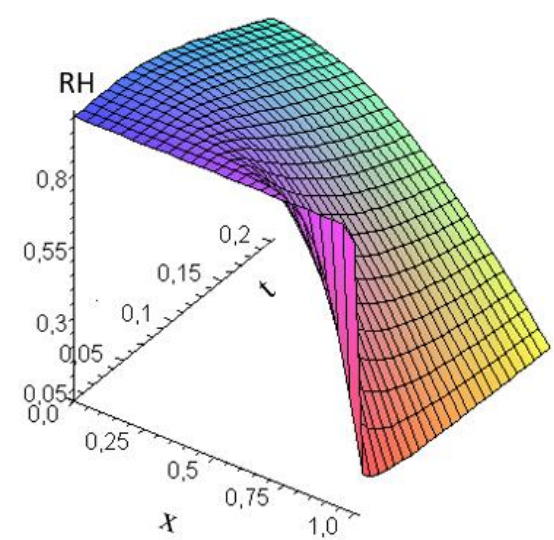

a)

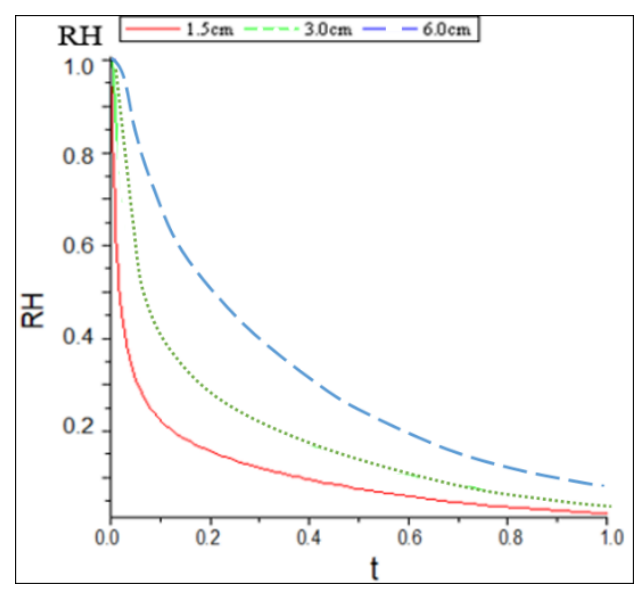

b)

Fig.2. Analytical solution for dimensionless humidity versus dimensionless time and space in 3D a); and RH time evolution at different positions to drying surface $b$ ).

\section{Drying model}

The drying model is fundamental for simulating the drying phenomenon in porous medium, and quite a few of literatures can be found in previous researches [11, 12]. For the drying model, it is assumed that the water transport in the concrete induced from the combination of the diffusion of water vapour and the permeation of liquid water. If the concrete porous network is saturated, the latter mechanism plays predominant role, whereas for a low degree of saturation, the movement of water in the form of vapour diffusion cannot be neglected. The simplified approach involves water transfer coefficient 
that is a function of saturation degree or the specific water $[13,14]$. The liquid water flow can be expressed by Darcy's law, which relates the fluid mass flow through the medium to the pressure gradient of this fluid. The water vapour flow can be expressed by Fick's law, representing the flow of water in form of vapour due to a gradient in vapour pressure in the considered porous area. The two driving forces expressed as pressure gradient, could be related to the relative humidity by Kelvin equation. The relation between the capillary forces and the relative humidity in concrete is expressed in Eq (4),

$$
p_{c}=\frac{-\rho_{l} R T \ln (H R)}{M_{w}}
$$

Deducing from the mass balance of water and the equilibrium driving forces, the final drying model is given in Eq. (5).

$$
\rho_{l}\left[\Phi \frac{\partial S_{l}}{\partial H R}\left(1-\frac{\rho_{v}}{\rho_{l}}\right)\right] \frac{\partial H R}{\partial t}=\operatorname{div}\left[\left(k_{l a} \frac{1}{H R} \frac{\rho_{l}^{2} R T}{\mu_{l} M_{v}}+D_{\text {eff }} \frac{M_{v} p_{v s}}{R T}\right) \operatorname{grad}(H R)\right]
$$

Among which, the desorption isotherm on the lefthand of the equation (5) links the saturation degree $\left(S_{l}\right)$ to the relative humidity and contribute to the water storing capacity. The most widely used expression is given by Eq. (6) [15], where $a_{v g}$ and $b_{v g}$ are two parameters that can be obtained by fitting experimental results of the desorption isotherm for the tested material.

$$
S_{l}=\left(1+\left(-a_{v g} \ln (H R)\right)^{b_{v g}}\right)^{-c_{v g}}
$$

On the right hand side of the Eq. (5), it suggests that the diffusion coefficient term (or hydrate conductivity) is associated with temperature, both for liquid permeation and vapour diffusion term. $k_{l a}$ is the apparent permeability of liquid, which can be expressed by intrinsic water permeability $k_{0}$ multiplied by relative permeability $k_{r l}\left(0<k_{r l}<1\right)$. This latter term can be determined by the Mualem empirical relation shown in Eq. (8), where $S_{l}$ is the saturation degree, $p_{k r l}$ is a fitting parameter [11].

$$
\begin{gathered}
k_{l a}=k_{r l} \cdot k_{0} \\
k_{r l}=S_{l}{ }^{p_{k r l}}\left(1-\left(1-S_{l}^{\frac{1}{a_{v g}}}\right)^{a_{v g}}\right)^{2}
\end{gathered}
$$

For the diffusion coefficient of vapour, the effective diffusivity $D_{\text {eff }}$ can be expressed by Eq. (9) and (10) [16].

$$
\begin{gathered}
D_{e f f}=d_{r l} D_{0} \\
d_{r l}=\Phi^{a_{m q}}\left(1-S_{l}\right)^{b_{m q}}
\end{gathered}
$$

The apparent diffusion is related to the relative diffusion function $d_{r l}$ and to the diffusion coefficient of water in air $D_{0}$. In a similar way, the diffusion of water vapour is highly dependent on the saturation degree. In addition, the tortuosity of the concrete porosity also affects these parameter $a_{m q}$ and $b_{m q}$.
What is worth noticing is that in the drying model, some parameters are not only function of humidity, but also function of temperature. In Eq. (5), despite of the apparent temperature, there is some other parameters affected by temperatures. The following Eqs. (11-13) illustrate the dependency to temperature, for the diffusion coefficient of water in air $D_{0}$, the saturation vapor pressure $P_{v s}$, and the dynamic viscosity of liquid water $\mu_{l}$. This also indicates the complex coupling between moisture and heat transfer, as well as the identification of parameters affecting the thermal property and hydrate property.

$$
\begin{gathered}
D_{0}=2.17 \times 10^{-5} \cdot \frac{P_{a t m}}{P_{g}} \cdot\left(\frac{T}{T_{0}}\right)^{2.88} \\
P_{v s}=P_{a t m} \cdot e^{\frac{M_{v} \cdot L_{v}}{R}\left(\frac{1}{T_{0}}-\frac{1}{T}\right)} \\
\mu_{l}=\mu_{0} \cdot e^{-1.94-4.8 \frac{T_{0}}{T}+6.74\left(\frac{T_{0}}{T}\right)^{2}}
\end{gathered}
$$

\section{Results and discussion}

Computational results of the drying model and the experimental results are compared in Fig. 3. Both the relative humidity evolution at the point of $1.5 \mathrm{~cm}$ (to drying surface) and relative mass loss (ML) are presented. The relative humidity provide a local information of the moisture distribution, while the mass loss is the integral of water content over the domain, meaning the global water loss during the drying process. The local humidity exhibit the decrease over time as consequence of the applied boundary condition of $32 \%$. This evolution has the same tendency as what was presented in the previous linear-simplified approach in Fig. 2b. During the test duration of 160 days, the simulation results Eqs. (5-12) match well with the experiment data using all the identified properties of the different contributing phenomenon.

In comparison to the first linear simplified case, this numerical model, which is more close to the reality, uses a variable volumetric capacity in the transient term and variable hydrate conductivity (humidity and water diffusion). We illustrate in the figures below (Fig. 4) the profile of the hydrate capacity and hydrate conductivity versus relative humidity in the present drying model.

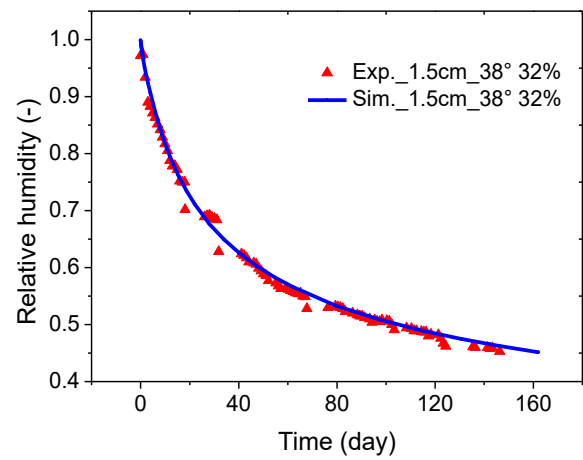

a) 


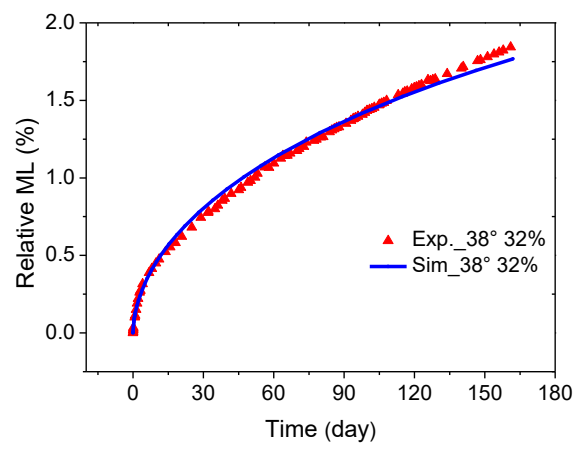

b)

Fig. 3. Experimental and simulation results of mass loss a); and relative humidity evolution $b$ ).

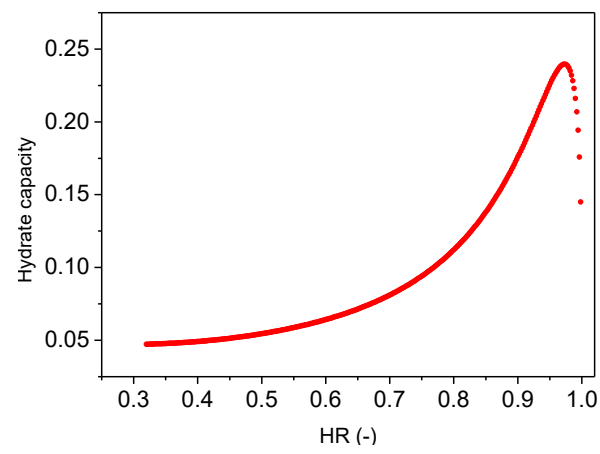

a)

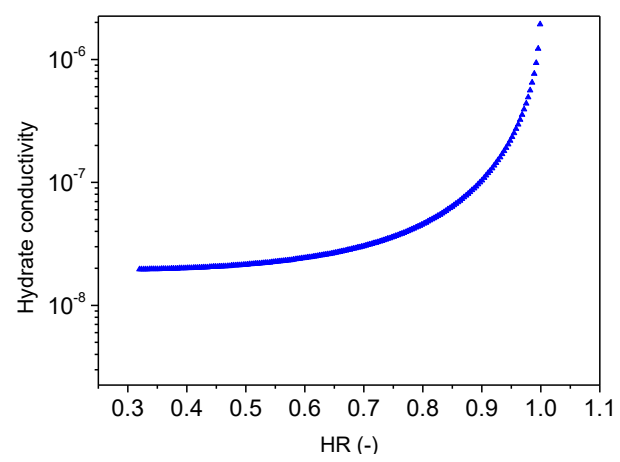

b)

Fig.4. Profile of equivalent hydrate capacity a); hydrate conductivity b) versus RH.

It demonstrates that both of the parameters are highly dependent to the water content level. This is obtained under the assumption of isotherm process, but it is worth noting that the humidity has effect on thermal properties as well, inducing the heat and mass transfer process.

\section{Conclusion}

This work emphasized on the hydrate transfer properties, and it is fundamental and necessary for accurately estimating the real local density, heat capacity, thermal conductivity and the heat source term in the heat transfer equation. The analysis can be applied to optimizing the hydrate and thermal properties and in the long-term predicting the durability of the cementitious materials.
This information also allows to resolve the drying model and to predict the temperature history and the exchange between the wall and the living area (air). In further level, it will be able to predict the durability of the inner structure and cracks within the material, with taking into account the change in properties and especially the permeability (water redistribution).

In short, the practical meaning of predicting the real distribution of hydrate state over space and time, including the evaporation or condensation, is to estimate accurately the real local density, heat capacity and thermal conductivity, as well as the source term in the heat transfer equation.

\section{Acknowledgments}

This ThermaSMART project has received funding from the European Union's Horizon 2020 -MSCA-RISE-2017 program under grant agreement No. 778104.

The first author acknowledges China Scholarship Council for the financial support, No. 201608120052.

\section{References}

1. H. Wang and J.E. Gillott, Cem. and Concr. Res., 21 647-654 (1991)

2. Z. Pavlik, and P. Rovnanikova, Int. J. Thermophys, 27 (4), 1228-1240 (2006)

3. M. Dell'Isola , F. R. D'Ambrosio Alfano, G. Giovinco, and E. Ianniello, Int. J. Thermophys, 33 (8-9), 1674-85 (2012)

4. K. Kim, J. Sang-eun, K. Jin-keun, and Y.Sungchul,. Cem. Concr. Res. 33, 363-71 (2003)

5. K. L. Bristow, Agricultural and Forest Meteorology 89 (2), 75-84 (1998)

6. D. Hillel, Fundamentals of Soil Physics. Academic Press, New York (1980)

7. S.G.S. Beirão, A.P.C. Ribeiro, M.J.V. Lourenço, F.J.V. Santos, and C.A. Nieto de Castro, Int. J. Thermophys. 33, 8-9 (2012)

8. D. Bednarska , and K. Marcin, AIP Conf. Proc. (June), 1577-78 (2017).

9. A. Hilaire, PhD Thesis, ENS Cachan (2014)

10. X. Ma, A. Darquennes, F. Benboudjema, G. Nahas, R. Bennacer, Int. Conf. Mater. Energy, (2017)

11. Y. Mualem, Water Resour. Res., 12(3), 513-522 (1976)

12. Z. Zhang, M. Thiery, Baroghel-Bouny, Cem. and Concr. Res., 89, 257-268 (2016)

13. Z. Bažant, L. Najjar, Cem. and Concr. Res., 1(5), 461-473 (1971)

14. B. Perrin, V. B. Bouny, L. Chemloul, Mater. Struct., 31(4), 235-241 (1998)

15. M. A. van Genuchten, Soil Science Society of America Journal, 44(5), 892-898 (1980)

16. R. Millington, J. Quirk, Trans. Faraday Soc., 57,1200-1207 (1961) 